\title{
REDUÇÃO DO CONSUMO DE ENERGIA DE CIRCUITOS DE MOAGEM COM A UTILIZAÇÃO DE PRÉ-CONCENTRAÇÃO DE MINERAIS SULFETADOS
}

\author{
M. B. BERGERMAN ${ }^{1}$, D. NETO ${ }^{1}$, B.Y. TOMASELLI ${ }^{2}$, B.F. MACIEL ${ }^{2}$, C. DEL ROVERI ${ }^{1}$, F.C. NAVARRO ${ }^{1}$ \\ ${ }^{1}$ Instituto de Ciência e Tecnologia - Universidade Federal de Alfenas \\ ${ }^{2}$ Votorantim Metais \\ mauricio.bergerman@unifal-mg.edu.br
}

Artigo submetido em novembro/2013 e aceito em dezembro/2013

DOI: http://dx.doi.org/10.15628/holos.2014.1798

\section{RESUMO}

O uso de operações mais complexas de cominuição e concentração de minerais na indústria mineral têm crescido, em função da queda dos teores e maior complexidade dos minérios disponíveis. Uma alternativa para enfrentar a alta dos custos com a instalação e operação de usinas de beneficiamento mineral é a préconcentração, usando-se métodos gravimétricos de beneficiamento. Com isso, descarta-se, antes da alimentação da usina de beneficiamento, uma fração com pouca ou nenhuma quantidade do mineral de interesse, reduzindo assim a massa a ser processada nas operações seguintes. Os benefícios gerados pelo uso da pré-concentração por meio de beneficiamento gravimétrico são significativos, garantindo, por exemplo, o aumento da produção de metal contido de uma usina sem necessidade de se recorrer a uma extensão da capacidade de moagem ou flotação e a extensão da vida útil da mina, devido à redução do teor de corte. O objetivo principal deste trabalho é avaliar o potencial de redução dos circuitos de cominuição de minerais sulfetados provenientes de um depósito de zinco, chumbo e cobre, utilizando a metodologia de préconcentração em meio denso. Foram desenvolvidos ensaios em escala laboratorial, buscando assim o aumento do teor dos metais de interesse que alimentam a moagem e a avaliação do WI de alimentação da usina.

PALAVRAS-CHAVE: Pré-concentração; Beneficiamento gravimétrico; Zinco; Sulfeto.

\section{SULPHIDE ORES GRINDING CIRCUITS ENERGY CONSUMPTION REDUCTION WITH PRE-CONCENTRATION}

\begin{abstract}
The use of more complex ore comminution and concentration operations in the mining industry has been growing in the recent years, due to the decrease of ore grades and increase complexity of ores. One of the alternatives to deal with high capital and operational costs of a mineral processing plant is the use of the preconcentration technology, using gravity concentration methods. With the use of such technology, it can be discarded, before the processing plant, a fraction of the ROM with less quantity of the interest minerals, thus
\end{abstract}

reducing the mass of ore that will feed the plant. Significant benefits can be obtained from the use of such a technology, as for example the increase at the plant metal production without the need to increase the grinding and flotation capacities. The main objective of this work is to evaluate the pre-concentration technology to concentrate a zinc, lead and copper sulphide ore. It was also evaluated the difference in the work index of the pre-concentrated feed.

KEYWORDS: Pre-concentration; Gravity concentration; Zinc, Sulphide. 


\section{INTRODUÇÃO}

O uso da operação de concentração de minerais na indústria mineral tem crescido, em função da queda dos teores e maior complexidade dos minérios disponíveis. Uma alternativa para enfrentar a alta dos custos com a instalação e operação de usinas de beneficiamento mineral é a pré-concentração. Para tal fim, podem ser utilizados métodos gravimétricos de beneficiamento, mais especificamente os métodos de separação em meio denso, como os separadores de cone e tambor Wenco, Teska, Drewboy, Norwalt, Dynawhirlpool, Tri-flo e os ciclones de meio denso (CAMPOS, LUZ e BRAGA, 2010). A escolha de cada um dos separadores é função principalmente da granulometria do material, vazões necessárias e faixa de densidades a se trabalhar. Mills (1980) ilustra os principais processos de separação gravimétrica e suas faixas recomendadas de aplicação, em função da quantidade de material próximo a densidade de separação, conforme mostrado na Tabela 1.

Tabela 1. Seleção dos métodos de concentração gravimétrica em função da quantidade de material próxima a densidade de separação (Adaptado de Mills, 1980)

\begin{tabular}{|c|c|c|c|}
\hline $\begin{array}{l}\text { \%mássica dentro de } \pm 0.10 \\
\text { densidade de separação }\end{array}$ & $\begin{array}{c}\text { Grau de dificuldade } \\
\text { esperado }\end{array}$ & $\begin{array}{l}\text { Processo gravimétrico } \\
\text { recomendado }\end{array}$ & Tipo \\
\hline $0-7$ & simples & quase qualquer processo & \multirow{3}{*}{$\begin{array}{c}\text { Jigues, mesas, } \\
\text { espirais, } \\
\text { comportas, cones, } \\
\text { vanners etc. }\end{array}$} \\
\hline $7-10$ & dificuldade moderada & processos eficientes & \\
\hline $10-15$ & difícil & $\begin{array}{c}\text { processos eficientes - boa } \\
\text { operação }\end{array}$ & \\
\hline $15-20$ & muito difícil & \multirow{2}{*}{$\begin{array}{l}\text { processos muito eficientes - } \\
\text { operação especializada }\end{array}$} & \multirow{2}{*}{$\begin{array}{l}\text { DMS (Dense Media } \\
\text { Separation) }\end{array}$} \\
\hline $20-25$ & extremamente difícil & & \\
\hline Acima de 25 & extremamente difícil & $\begin{array}{l}\text { limitado a poucos processos } \\
\text { excepcionalmente eficientes- } \\
\text { operação especializada }\end{array}$ & $\begin{array}{l}\text { DMS com controle } \\
\text { fechado }\end{array}$ \\
\hline
\end{tabular}

Com o uso destas tecnologias, descarta-se, antes da alimentação da usina de beneficiamento, uma fração com pouca ou nenhuma quantidade do mineral de interesse, reduzindo assim a massa a ser processada nas operações seguintes. Isso permite a redução de custos de capital e operacionais, além de minimizar o impacto ambiental dos projetos. Klein et al.(2010) preveem uma economia aproximada de $20 \%$ no consumo total de energia de projetos que utilizem etapas de pré-concentração.

Esta tecnologia já vem sendo amplamente aplicada nas minas de sulfetos como cobre, níquel, chumbo e zinco da África, da América do Norte e Austrália.

A mina Tati Niquel Phoenix Mine (MORGAN, 2009), localizada em Botswana, reporta a instalação de uma etapa de britagem e pré-concentração com ciclones de meio denso com capacidade para $1.600 \mathrm{t} / \mathrm{h}$, processando material entre $25 \mathrm{~mm}$ e $1 \mathrm{~mm}$. Esta instalação permitiu o descarte de $60 \%$ do minério proveniente da mina. Devido à mudança nas características de alimentação do circuito de moagem, com utilização da pré-concentração, um ganho adicional foi 
obtido, com o aumento da capacidade do circuito de moagem em 40\%, para $650 \mathrm{t} / \mathrm{h}$. Para subsidiar a instalação desta operação industrial, foram realizados ensaios de separação em meio denso em escala laboratorial e foi operada uma planta piloto com capacidade para $200 \mathrm{t} / \mathrm{h}$ nas instalações da Mintek, na África do Sul. Dados da operação industrial de Tati (DENYSSCHEN e WAGNER, 2009) indicam que atualmente o minério com teor acima de 0,56\% de níquel é alimentado diretamente no circuito de moagem e flotação. O material que alimenta a planta de DMS apresenta um teor de 0,31 a 0,37\%. Após o peneiramento e segregação da fração abaixo de $1 \mathrm{~mm}$, este teor cai para 0,27 a $0,32 \%$ de níquel. O concentrado da planta de DMS apresenta um teor entre 0,41 a $0,50 \%$ de níquel e o rejeito entre 0,07 e $0,09 \%$ de níquel.

Fourie et al. (2007), apresenta o resultado da avaliação do uso do DMS na mina Rosh Pinah Zinc Corporation na Namíbia. Foi avaliada a pré-concentração de minério proveniente de uma mina subterrânea, britado e classificado entre 32 e $16 \mathrm{~mm}$, com um teor de zinco de aproximadamente $3 \%$. O concentrado teria $9 \%$ de zinco. Para uma densidade de $2,85 \mathrm{t} / \mathrm{m} 3$, foram obtidas recuperações metalúrgicas da ordem de $90 \%$, com uma recuperação em massa de $30 \%$. O uso da tecnologia de pré-concentração com ore sorters com sensores de Raios- $X$ também está sendo avaliada. O plano da empresa é instalar a pré-concentração em subsolo, evitando assim o transporte do material já descartado a superfície.

Creswell (2001) cita o grande potencial de aplicação desta tecnologia e ilustra uma série de resultados positivos em minas da África, México e China. O autor apresenta recuperações metalúrgicas da ordem de 90 a 95\% dos metais de interesse com o descarte de 15 a 30\% da massa que alimentaria a usina.

No Brasil, esta tecnologia já foi utilizada na mina de fluorita da Nitroquímica, paralisada desde 2009.

Os benefícios gerados pelo uso da pré-concentração por meio de beneficiamento gravimétrico são significativos, garantindo, por exemplo, o aumento da produção de metal contido de uma usina sem necessidade de se recorrer a uma extensão da capacidade de moagem ou flotação e a extensão da vida útil da mina, devido à redução do teor de corte. Ganhos adicionais podem ser obtidos com a rejeição da ganga antes do processo de moagem, já que algumas vezes esta possui um WI de Bond maior que o dos minerais de minério.

O objetivo principal deste trabalho é avaliar a variação do Work Index de Bond (WI) em duas amostras de minério sulfetado de zinco e chumbo após a sua concentração gravimétrica, simulando um processo de DMS. Tendo em vista as pequenas quantidades de massas disponíveis para determinação do WI devido a escala dos ensaios de separação em meio denso, foi utilizada para a determinação deste a metodologia simplificada proposta por Jauregui (1982).

\section{MATERIAIS E MÉTODOS}

O trabalho experimental foi dividido em três etapas. Na primeira, as amostras foram compostas, britadas e peneiradas. Na segunda etapa a amostra foi separada, testando-se diferentes granulometrias e densidades. Na densidade e granulometria considerada ótima, uma maior alíquota foi separada, visando a geração de massa para os ensaios de moagem. Na terceira etapa, foram realizados ensaios simplificados para a determinação do WI nas amostras geradas. $O$ procedimento experimental detalhado é apresentado a seguir. 


\subsection{Composição das amostras, britagem e peneiramento}

Inicialmente as amostras foram britadas em um britador de mandíbulas. Após a primeira passagem no britador de mandíbulas, a amostra foi classificada utilizando-se uma peneira de malha quadrada de $12,7 \mathrm{~mm}$ de abertura. O produto retido em $12,7 \mathrm{~mm}$ foi britado novamente, sendo o retido recirculado neste britador até que a quase totalidade do material fosse passante nesta malha.

O produto da britagem foi então homogeneizado e quarteado, utilizando o método de pilha alongada. Foi retirada uma alíquota de aproximadamente $500 \mathrm{~g}$ para análise química e outra de 2 $\mathrm{kg}$ para análise granulométrica por peneiramento a seco, utilizando-se peneiras com aberturas de $12,7 \mathrm{~mm}, 9,5 \mathrm{~mm}, 6,35 \mathrm{~mm}, 4,75 \mathrm{~mm}, 3,35 \mathrm{~mm}, 2,36 \mathrm{~mm}, 1,68 \mathrm{~mm}, 1,17 \mathrm{~mm}, 0,85 \mathrm{~mm}, 0,60 \mathrm{~mm}$ e $0,425 \mathrm{~mm}$. Os produtos obtidos no peneiramento foram quarteados e enviados para análise química. $O$ restante do material foi separado em alíquotas de aproximadamente $10 \mathrm{~kg}$.

\subsection{Ensaios de separação mineral em líquido denso}

A partir dos resultados de análises químicas por fração granulométrica da etapa anterior, foram compostas 3 frações granulométricas para os ensaios de separação mineral em líquido denso, sendo a primeira acima de $6,3 \mathrm{~mm}$, a segunda entre $6,3 \mathrm{~mm}$ e $3,35 \mathrm{~mm}$ e a terceira entre $3,35 \mathrm{~mm}$ e 1,18 mm. A fração fina, abaixo de 1,18 mm, foi descartada, por ser muito fina para separações por métodos densitários com as tecnologias planejadas para uso industrial.

Cada fração descrita acima foi separada em três densidades diferentes, conforme ilustrado na Tabela 2.

Tabela 2. Reagentes utilizados para o ensaio de líquido denso

\begin{tabular}{c|c|c}
\hline Reagente & Fórmula química & Densidade $\left(\mathrm{t} / \mathrm{m}^{3}\right)$ \\
\hline Tetrabromoetano puro & $\mathrm{CHBr}_{2} \mathrm{CHBr}_{2}$ & 2,96 \\
\hline Tribromoetano puro (bromofórmio) & $\mathrm{CHBr}_{3}$ & 2,89 \\
\hline Tribromoetano diluído com álcool etílico (Etanol) & $\mathrm{CH}_{3} \mathrm{CH}_{2} \mathrm{OH}$. & 2,76 \\
\hline
\end{tabular}

A seleção dos líquidos densos e respectivos diluentes a serem utilizados, assim como os cuidados a serem tomados durante os ensaios foram baseados nos textos de Burt (1984) e Sampaio e Tavares (2005).

A densidade do líquido denso, quando diluída com álcool, era verificada a cada ensaio, com um balão volumétrico de $200 \mathrm{~mL}$, pesando-se o mesmo, sendo corrigida caso necessário. Ao todo, para cada amostra composta, foram realizados 9 ensaios de separação em líquido denso (três densidades em três frações granulométricas). As amostras geradas nos ensaios de meio denso foram encaminhadas para análises químicas.

\subsection{Ensaio simplificado para a determinação do WI}

O ensaio para determinação do WI foi realizado seguindo a metodologia simplificada proposta por Jauregui (1982). O mesmo é realizado em moinho de bancada em circuito aberto, 
que tenha sido previamente calibrado com diversos minérios em comparação ao moinho de Wl. 0 autor assume que se a mesma distribuição granulométrica de alimentação for usada para diferentes minérios submetidos a uma mesma energia de moagem, uma competência a moagem relativa pode ser determinada, em função das diferentes distribuições granulométricas obtidas para o produto. O ensaio é realizado com uma amostra de $1 \mathrm{~kg}$, britada abaixo de 1,68 $\mathrm{mm}$ e sem a fração abaixo de 0,150 mm, cominuída em um moinho de $21 \times 25,1 \mathrm{~cm}$, com corpos moedores entre 3,81 e 2,22 cm, com $92 \mathrm{rpm}$ e $50 \%$ de sólidos por 10 minutos. O produto de cada ensaio de moagem foi peneirado a úmido. A distribuição da carga de bolas utilizada foi similar listada abaixo (YAP, SEPULVEDA E JAREGUI, 1982):

$$
\begin{aligned}
& \text { 3,81-3,56 cm (1,5 a 1,4 in })-11 \text { bolas }-3261,5 \mathrm{~g} \\
& 3,30-3,18 \mathrm{~cm}(1,30 \text { a 1,25 in) }-17 \text { bolas }-2325,4 \mathrm{~g} \\
& 3,10-2,92 \mathrm{~cm}(1,22 \text { a 1,15 in) }-13 \text { bolas }-1534,8 \mathrm{~g} \\
& 2,79-2,54 \mathrm{~cm}(1,10 \text { a 1,00 in) }-10 \text { bolas }-822,5 \mathrm{~g} \\
& 2,54-2,41 \mathrm{~cm}(1,00 \text { a } 0,95 \mathrm{in})-7 \text { bolas }-449,7 \mathrm{~g} \\
& 2,41-2,29 \mathrm{~cm}(0,95 \text { a } 0,90 \mathrm{in})-30 \text { bolas }-1634,0 \mathrm{~g}
\end{aligned}
$$

Tendo em vista que foram utilizadas neste ensaio as mesmas condições propostas por Jauregui (1982), a constante de calibração utilizada foi a mesma apresentada pelo autor, de 0,5031. Deste modo, o WI da amostra estudada é calculada a partir da Equação 1.

$$
W I=K *\left(\frac{1}{\sqrt{P}}-\frac{1}{\sqrt{A}}\right)
$$

Jauregui (1982) apresenta um erro de aproximadamente $5 \%$ entre os valores medidos pelo WI e pelo método simplificado.

\section{RESULTADOS E DISCUSSÃO}

O resultado da análise química das duas amostras estudadas é apresentado na Tabela 3.

Tabela 3. Teores das amostras recebidas após composição

\begin{tabular}{c|c|c}
\hline Teor & Amostra 1 - Disseminado & Amostra 2 - Stringer \\
\hline $\mathrm{Zn} \mathrm{( \% )}$ & 0,585 & 2,37 \\
\hline $\mathrm{Pb} \mathrm{( \% )}$ & 1,07 & 2,50 \\
\hline $\mathrm{Cu}(\mathrm{ppm})$ & 162 & 693 \\
\hline $\mathrm{Ag} \mathrm{(ppm)}$ & 5,4 & 7,8 \\
\hline $\mathrm{S}(\%)$ & 1,41 & 2,52 \\
\hline
\end{tabular}

A mineralização se encontra principalmente em uma matriz de arenito. 
Os dados experimentais de granulometria e química por faixa mostram que os elementos de interesse se distribuem de maneira bastante homogênea em todas as faixas granulométricas, com distribuições proporcionais a massa de cada fração.

Os resultados dos ensaios de separação em líquido denso para a amostra 2, do minério Stringer, são ilustrados na Tabela 4.

Tabela 4. Resultados dos ensaios exploratórios de líquido denso - Amostra 2 - Stringer

\begin{tabular}{|c|c|c|c|c|c|c|c|c|c|c|c|c|c|c|c|c|c|c|}
\hline \multirow{3}{*}{ Fração $(\mathrm{mm})$} & \multirow{3}{*}{ Produto } & \multirow{2}{*}{\multicolumn{2}{|c|}{ Massa (\%) }} & \multicolumn{5}{|c|}{ Teores } & \multicolumn{5}{|c|}{ Distribuição ensaio (\%) } & \multicolumn{5}{|c|}{ Distribuição amostra (\%) } \\
\hline & & & & $\mathrm{Zn}$ & $\mathrm{Pb}$ & $\mathrm{Cu}$ & $\mathrm{Ag}$ & $\mathrm{s}$ & $\mathrm{Zn}$ & $\mathrm{Pb}$ & $\mathrm{Cu}$ & $\mathrm{Ag}$ & $\mathrm{s}$ & $\mathrm{Zn}$ & $\mathrm{Pb}$ & $\mathrm{Cu}$ & $\mathrm{Ag}$ & s \\
\hline & & Ensaio & Amostra & $\%$ & $\%$ & $\%$ & $\mathrm{ppm}$ & $\%$ & & & & & & & & & & \\
\hline \multirow{6}{*}{$+6,3$} & Afundado 2,96 & 6,7 & 2,1 & 23,10 & 8,27 & 0,448 & 32,00 & 15,00 & $\begin{array}{ll}69,7 \\
\end{array}$ & 22,6 & 59,0 & 30,0 & 45,0 & 20,6 & 7,0 & 13,7 & 8,7 & 12,6 \\
\hline & Afundado 2,86 & 1,6 & 0,5 & 10,85 & 6,41 & 0,120 & 15,90 & 6,49 & 8,0 & 4,3 & 3,9 & 3,6 & 4,8 & 2,4 & 1,3 & 0,9 & 1,1 & 1,3 \\
\hline & Afundado 2,75 & 1,2 & 0,4 & 6,26 & 5,76 & 0,816 & 53,00 & 4,68 & 3,5 & 2,9 & 19,8 & 9,1 & 2,6 & 1,0 & 0,9 & 4,6 & 2,6 & 0,7 \\
\hline & Flutuado & 90,5 & 28,7 & 0,46 & 1,89 & 0,010 & 4,50 & 1,17 & 18,9 & 70,2 & 17,3 & 57,2 & 47,6 & 5,6 & 21,7 & 4,0 & 16,5 & 13,3 \\
\hline & Total calculado & 100,0 & 31,7 & 2,21 & 2,44 & 0,051 & 7,11 & 2,22 & 100,0 & 100,0 & 100,0 & 100,0 & 100,0 & 29,6 & 30,9 & 23,2 & 28,9 & 28,0 \\
\hline & Total analisado* & & & 2,00 & 2,59 & 0,141 & 10,56 & 2,29 & & & & & & & & & & \\
\hline \multirow{5}{*}{$-6,3+3,35$} & Afundado 2,96 & 8,7 & 2,8 & 31,34 & 10,10 & 0,318 & 30,30 & 18,05 & 80,3 & 32,4 & 39,7 & 33,2 & 54,1 & 37,0 & 11,3 & 12,8 & 10,9 & 20,0 \\
\hline & \begin{tabular}{|l} 
Afundado 2,86 \\
Afundado 2,75
\end{tabular} & $\begin{array}{l}2,6 \\
3,8\end{array}$ & $\begin{array}{l}0,8 \\
1,2\end{array}$ & 4,43 & 6,62 & 0,474 & 27,00 & 4,45 & 8,4 & 15,8 & 43,9 & 21,9 & 9,9 & 3,9 & 5,5 & 14,2 & 7,2 & 3,7 \\
\hline & Flutuado & 84,9 & 27,3 & 0,45 & 1,66 & 0,013 & 4,20 & 1,23 & 11,3 & 51,8 & 16,3 & 44,9 & 36,0 & 5,2 & 18,1 & 5,3 & 14,7 & 13,3 \\
\hline & Total calculado & 100,0 & 32,1 & 3,40 & 2,71 & 0,070 & 7,94 & 2,90 & 100,0 & 100,0 & 100,0 & 100,0 & 100,0 & 46,0 & 34,8 & 32,3 & 32,7 & 37,0 \\
\hline & Total analisado* & & & 2,62 & 2,66 & 0,034 & 6,57 & 2,58 & & & & & & & & & & \\
\hline \multirow{6}{*}{$-3,35+1,18$} & Afundado 2,96 & 7,8 & 1,5 & & & & & & & & & & & & & & & \\
\hline & Afundado 2,86 & 1,9 & 0,4 & 18,95 & 9,01 & 0,652 & 45,30 & 12,80 & 85,7 & 39,3 & 84,6 & 55,6 & 56,0 & 17,1 & 7,7 & 20,1 & 12,4 & 10,9 \\
\hline & Afundado 2,75 & 1,1 & 0,2 & & & & & & & & & & & & & & & \\
\hline & Flutuado & 89,2 & 17,6 & 0,39 & 1,69 & 0,014 & 4,40 & 1,22 & 14,3 & 60,7 & 15,4 & 44,4 & 44,0 & 2,9 & 11,9 & 3,7 & 9,9 & 8,5 \\
\hline & Total calculado & 100,0 & 19,7 & 2,40 & 2,48 & 0,083 & 8,83 & 2,47 & 100,0 & 100,0 & 100,0 & 100,0 & 100,0 & 20,0 & 19,6 & 23,8 & 22,3 & 19,4 \\
\hline & Total analisado* & & & 2,49 & 2,51 & 0,114 & 9,36 & 2,51 & & & & & & & & & & \\
\hline \multirow{6}{*}{ Total $+1,18$} & \begin{tabular}{|l|} 
Afundado 2,96 \\
\end{tabular} & 7,7 & 6,4 & 25,69 & 9,24 & 0,440 & 34,43 & 15,80 & 74,9 & 29,0 & 57,8 & 35,7 & 49,2 & 74,7 & 26,0 & 46,6 & 32,0 & 43,5 \\
\hline & Afundado 2,86 & 2,1 & 1,7 & 9,53 & 7,08 & 0,408 & 27,74 & 6,89 & 7,5 & 6,0 & 14,5 & 7,8 & 5,8 & 6,2 & 6,8 & 15,1 & 8,2 & 5,0 \\
\hline & Afundado 2,75 & 2,2 & 1,8 & 3,59 & 2,29 & 0,250 & 16,62 & 2,52 & 3,0 & 2,1 & 9,4 & 4,9 & 2,2 & 1,0 & 0,9 & 4,6 & 2,6 & 0,7 \\
\hline & Flutuado & 88,0 & 73,6 & 0,44 & 1,75 & 0,012 & 4,36 & 1,20 & 14,6 & 62,9 & 18,3 & 51,6 & 42,8 & 13,7 & 51,6 & 12,9 & 41,2 & 35,1 \\
\hline & Total calculado & 100,0 & 83,6 & 2,65 & 2,46 & 0,059 & 7,44 & 2,48 & 100,0 & 100,0 & 100,0 & 100,0 & 100,0 & 95,6 & 85,3 & 79,2 & 84,0 & 84,3 \\
\hline & Total analisado* & & & 2,29 & 2,60 & 0,101 & 9,08 & 2,43 & & & & & & & & & & \\
\hline Cabeça & & & 100 & 2,37 & 2,50 & 0,069 & 7,80 & 2,52 & & & & & & & & & & \\
\hline
\end{tabular}

A amostra Stringer apresentou uma clara tendência de melhora de recuperação para as frações mais finas. Deve-se ressaltar, no entanto, que mesmo na fração grosseira, os resultados obtidos foram muito positivos, tendo se observado um enriquecimento significativo dos elementos de interesse nas frações afundadas. Na fração acima de $6,5 \mathrm{~mm}$, o teor de Zinco do afundado em $2,96 \mathrm{~g} / \mathrm{cm} 3$ aumentou de $2,21 \%$ para $23,10 \%$. O teor de chumbo variou de $2,44 \%$ para $8,27 \%$ e o teor de prata subiu de 7,1 ppm para $32 \mathrm{ppm}$. Nesta mesma fração, apenas $7 \%$ da massa concentrou $70 \%$ do zinco da amostra, $23 \%$ do chumbo e $30 \%$ da prata. Os resultados indicam que mesmo nesta fração mais grosseira, caso sejam testadas densidades mais baixas de separação, ótimos resultados de recuperação metalúrgica com uma pequena recuperação em massa poderão ser obtidos. As demais frações granulométricas apresentaram resultados semelhantes em termos de enriquecimento e recuperação metalúrgica para baixos valores de recuperação em massa. Os resultados obtidos para a amostra 1 , de minério disseminado, apresentou resultados semelhantes. Em função das partições e recuperação obtidas, foi selecionada a granulometria mais grosseira, abaixo de $12,7 \mathrm{~mm}$, separada em uma densidade de $2,7 \mathrm{~g} / \mathrm{cm} 3$, para a realização dos ensaios de moagem. Estes resultados indicam que o uso do DMS pode significar uma significativa redução no consumo energético por tonelada de metal alimentado a usina

Os resultados dos ensaios de moagem são apresentados na Tabela 5. 
Tabela 5. Resultados dos ensaios de moagem

\begin{tabular}{c|c|c|c|c|c}
\hline Amostra & $\begin{array}{c}\text { Fração do ensaio } \\
\text { de líquido denso }\end{array}$ & $\begin{array}{c}\text { Constante de } \\
\text { calibração }(\mathrm{K})\end{array}$ & $\begin{array}{c}\mathrm{A}_{80} \\
(\mathrm{~mm})\end{array}$ & $\begin{array}{c}\mathrm{F}_{80} \\
(\mathrm{~mm})\end{array}$ & $\begin{array}{c}\text { WI* } \\
(\mathrm{kWh} / \mathrm{t})\end{array}$ \\
\hline \multirow{2}{*}{ 1- Disseminado } & Flutuada & 0,5031 & $1.452,2$ & 129,2 & 8,15 \\
\cline { 2 - 6 } & Afundada & 0,5031 & $1.485,5$ & 131,3 & 8,20 \\
\hline \multirow{2}{*}{2 - Stringer } & Flutuada & 0,5031 & $1.420,3$ & 138,8 & 8,65 \\
\cline { 2 - 6 } & Afundada & 0,5031 & $1.497,4$ & 134,9 & 8,47 \\
\hline
\end{tabular}

*Simplificado, calculado pelo método de Jauregui (1982).

Os resultados dos ensaios de moagem indicam um que a fração afundada da amostra 2 Stringer, apresenta um WI aproximadamente $2 \%$ menor que o da fração flutuada. No caso da amostra 1 - Disseminado, o WI da fração afundada é $0,65 \%$ maior que o da fração flutuada. Os resultados indicam que não ocorre uma diferenciação significativa do WI entre as frações afundadas e flutuadas.

Essa pequena diferença entre ambas frações pode se explicar pelo baixo $\mathrm{WI}$ da rocha na qual se encontra a mineralização dos sulfetos, um arenito, que assim como o sulfeto, possui um WI relativamente baixo.

\section{CONCLUSÕES}

O estudo de pré-concentração dos sulfetos da amostra estudada apresentou bons resultados frente aos ensaios de líquido denso realizados em escala de laboratório. Mesmo em produtos relativamente grosseiros, abaixo de $12,7 \mathrm{~mm}$, a amostra 2, do minério stringer, apresentou, para uma recuperação em massa de $7 \%$ no ensaio (considerando-se o acumulado do afundado na densidade 2,96 t/m3), uma recuperação de $70 \%$ do zinco da amostra, $23 \%$ do chumbo e $30 \%$ da prata. Para esta densidade, o teor de Zinco do afundado em 2,96 g/cm3 aumentou de $2,21 \%$ para $23,10 \%$. O teor de chumbo variou de $2,44 \%$ para $8,27 \%$ e o teor de prata subiu de 7,1 ppm para $32 \mathrm{ppm}$. Estes resultados indicam que o uso do DMS pode significar uma significativa redução no consumo energético por tonelada de metal alimentado a usina.

Os ensaios de moagem, realizados com os produtos dos ensaios de líquido denso das duas amostras não apresentou diferenças significativas de WI. Esse resultado se deve ao fato da mineralização se encontrar em um arenito, rocha que possui um WI relativamente baixo, não muito diferente do sulfeto que contém a mineralização. Espera-se que para amostras de minérios sulfetados cujas rochas sejam mais competentes, como granitos, os resultados do WI das frações enriquecidas em sulfetos sejam significativamente menores. Estudos em amostras com estas características já estão sendo realizados de forma a se confirmar tais hipóteses.

\section{AGRADECIMENTOS}

Os autores agradecem a Votorantim Metais pela autorização para publicação deste trabalho e a Fapemig e a Pró-reitoria de pesquisa e pós-graduação da Unifal-MG pelo apoio na apresentação do mesmo. 


\section{REFERÊNCIAS BIBLIOGRÁFICAS}

1. BURT, R.O. Gravity Concentration Technology. Amsterdam: Elsevier,1984.

2. CAMPOS, A.R.; LUZ, A.B.; BRAGA, P.F.A. Separação em meio denso. In: DA LUZ, A.B; SAMPAIO, J.A; FRANÇA, S.C.A. Tratamento de Minérios. Rio de janeiro: CETEM/MCT, 2010. 5a Ed. Cap. 8. P. 329-366.

3. CRESWELL, G.M. Pre-concentration of base metal ores by dense medium separation. In: Saimm Cooper, Cobalt, Nickel and Zinc Recovery Conference, 2001, Joanesburgo: SAIMM, 2001. P. 1-10.

4. DENYSSCHEN, D.F.; WAGNER, B.N. Pre-concentration of low grade lateritic sulphide nickel ore. In: Base Metals Conference, 2009, Kasane. Joanesburgo: SAIMM, 2009. P. 291-306.

5. FOURIE, H.; ROOYEN, P.H.V.; RUPPRECHT, S.; LUND, T.; VEGTER, N.M. Exploitation of a massive low grade zinc-lead resource at Rosh Pinah Zinc Corporation, Namibia. In: The Fourth Southern African Conference on Base Metals, 2007, Swakopmund. Joanesburgo: SAIMM, 2007. P. 109118.

6. JAUREGUI, R.O. Simplified Bond Work Index determination, In: Encontro nacional de tratamento de minérios, IX, 1982, Rio de Janeiro. Anais. Rio de Janeiro: Capes, 1982. p. $358-$ 367.

7. KLEIN, B. Energy efficienty technologies for the mining industry. In: Cim Conference, 2010, Vancouver. Westmount: CIM, 2010.

8. MILLS, C. Process design, scale-up, and plant design for gravity concentration. In: Mular, A.R.; Bhappu.R.B. Mineral Processing Plant Design. Littleton: SME, 1980. Cap. 18. P. 404-426.

9. MORGAN, P. The impact of a crushing plant upgrade and dms pre-concentration on the processing capability of the Tati Nickel Concentrator. In: Base Metals Conference, 2009, Kasane. Joanesburgo: SAIMM, 2009. P. 231-244.

10. SAMPAIO, C.H; TAVARES, L.M.M. Beneficiamento Gravimétrico. Porto Alegre: Editora da UFRGS, 2005.

11. YAP, R. F.; SEPULVEDA, J.L.; JAUREGUI, R. Determination of the Bond Work Index using an ordinary laboratory batch ball mill. In: Design and installation of comminution circuits. Edited by Mular, A. L. e Jergensen II, G.V. New York: Society of mining engineers. 1982. p. 176 a 203. 